\title{
LAS REDES FAMILIARES EN EL PROCESO MIGRATORIO DE LOS ECUATORIANOS A ESPAÑA
}

\author{
BLANCA PESANTEZ \\ CESPLA, Universidad de Cuenca (Ecuador).
}

\section{RESUMEN}

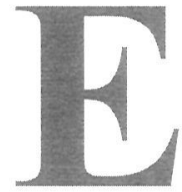

n la construcción de teorías sobre la migración han predominado aquéllas que rescatan la parte económica y los factores estructurales como determinantes de este proceso. Sin embargo, actualmente, se requiere prestar mayor atención a otros elementos que amplíen esta visión, como los tipos de familia, los sistemas de parentesco, los sistemas sociales y las estructuras sociales en general, así como a los aspectos y contextos culturales de la migración. La incorporación de estos elementos permitirá analizar este hecho social como un hecho complejo.

Palabras clave: migración, Ecuador, redes sociales, familias transnacionales.

\section{INTRODUCCIÓN}

Por la importancia que adoptan dentro del proceso migratorio las redes sociales, Ubaldo Martínez Veiga considera que deben ser abordadas como un organizador social de dicho proceso. Este concepto tiene una larga tradición que se remonta a Thomas y Znaniecki (Revista Internacional de Ciencias Sociales, 2000: 40).

Desde esta perspectiva, las redes migratorias se constituyen en una de las maneras de abordar la migración contemporánea, éstas pueden definirse como "un conjunto de relaciones interpersonales que vinculan a los migrantes o migrantes retornados con los parientes, amigos o compatriotas que permanecen en el país de origen" (ibid. 41).

En esta ocasión, se pretende considerar a las redes sociales como un elemento explicativo del crecimiento de los flujos migratorios de los ecuatorianos a España, desde finales de la década de los 90 hasta agosto del $2003^{\prime}$. En este artículo, se pondrá énfasis en las redes familiares porque su presencia es predominante en la emigración de los ecuatorianos.

Los datos utilizados para la elaboración de este trabajo forman parte de

1 A partir de esta fecha empieza a exigirse la Visa a los ecuatorianos que ingresan al territorio español. 
la investigación binacional: Ecuador/España, llevada a cabo por el equipo de investigadores del Centro de Estudios Sociales y Políticos Latinoamericanos -CESPLA- y por el Departamento de Sociología II de la Universidad de Alicante.

Con respecto a la metodología, en este proyecto de investigación se utilizó la "triangulación metodológica" porque favorece un conocimiento más amplio y profundo de la realidad social a investigarse. Se utilizaron algunos datos estadísticos oficiales de Ecuador y España sobre el tema, sin embargo, los datos en base a los cuales se construyen algunos planteamientos sobre las redes sociales fueron obtenidos por medio de la técnica cualitativa de los relatos cruzados. Esta técnica se constituye a partir del trabajo de investigación desarrollado en dos momentos: el primero con los familiares de los migrantes en Ecuador y el segundo con los migrantes en España.

A partir de los "relatos" obtenidos mediante las entrevistas a profundidad, se procedió a contrastar la información de las dos partes. Esta técnica enriquece el análisis respectivo porque proporciona una visión integral de cómo funcionan los procesos migratorios y sus consecuentes implicaciones, que tienen características propias si tomamos como referencia del análisis la posición - del familiar o del migrante- así como el lugar geográfico en el que se encuentran, país de origen o país de destino .

La información recopilada a través de esta técnica se obtuvo de 20 familias en Ecuador - de las provincias de Azuay, Cañar y Loja- y de 20 emigrantes en España -de Madrid, Barcelona, Murcia, Jumilla y Alicante-. En el caso de las entrevistas realizadas en Ecuador, la relación de parentesco con los emigrantes que se tomó como base es la de padres e hijos, hermanos, esposos. Para la selección de los entrevistados se tuvo en consideración algunas variables: lugar de origen, lugar de destino, tiempo de migración, situación, mercado laboral, instrucción ${ }^{2}$.

El objetivo general que guía el tratamiento de este tema es conocer las formas y funciones de las redes sociales de los ecuatorianos en España, así como su influencia en la persistencia o crecimiento de los flujos migratorios.

Los objetivos específicos son: a) relacionar los enfoques teóricos sobre las redes sociales con el funcionamiento de las mismas en el caso específico de los migrantes ecuatorianos en España y b) conocer cómo funcionan las dimensiones instrumentales de las redes y hasta qué punto éstas facilitan o dificultan la movilidad social.

\section{ENFOQUES TEÓRICOS}

\subsection{Las comunidades transnacionales}

Ante el aumento considerable de grupos que forman parte de circuitos migratorios internacionales y que se establecen en áreas geográficas determinadas generando un tipo especial de lazos sociales, se hace imprescindible utilizar nuevos términos que designen este tipo de acontecimientos. Hablar de

En el anexo de este artículo se presenta el perfil tipológico definitivo de los entrevistados. 
"comunidades transnacionales" significa abordar la migración desde un enfoque teórico, concebida ésta no como un cambio de la residencia habitual sino como un estado y forma de vida.

Según Smith, es factible hablar de comunidades transnacionales porque "mediante la migración se activarian diversos factores y procesos de articulación en los ámbitos cultural, social y económico, entre comunidades e instituciones sociales distantes geográficamente" (citado por Canales y Zlolniski, 2002: 414).

Desde esta perspectiva, "comunidad" no debe entenderse a partir de su concepto tradicional, que resalta las dimensiones temporales y espaciales, sino que la debemos entender en un contexto de "desterritorialización", es decir, como la expansión transnacional del espacio de las comunidades mediante las prácticas sociales, artefactos y sistemas simbólicos. Goldring define el transnacionalismo como "el proceso por el cual los migrantes construyen estos campos sociales que unen sus propias comunidades y sociedades de origen con las de asentamiento" (ibid. 421).

Desde este punto de vista, se considera que la "transnacionalidad" no es realmente un fenómeno nuevo. "Los migrantes económicos, que solían concebir la migración como algo temporal, mantenian espontáneamente vínculos con sus paises de origen. Lo nuevo en cuanto a la transnacionalidad es su organización: redes construidas y comunidades estructuradas" (Revista Internacional de Ciencias Sociales, 2000: 59).

Esta nueva organización a la que se hace referencia se ve facilitada por factores como los sistemas de redes y de relaciones sociales, las mismas que se van estructurando a través de la práctica. Alejandro Portes señala que "las comunidades transnacionales se fundan en un denso sistema de redes sociales que cruzan las fronteras politicas y que son creadas por los migrantes en su búsqueda de reconocimiento social y avance económico" (ibid. 240). Entonces, son las redes sociales las que proporcionarán los elementos que permitan el cruce de fronteras al que se refiere Portes, es decir, los vínculos de parentesco, amistad, el sentido de pertenencia a esas redes originadas por el carácter de identidad comunitaria, etc.

Como producto de las comunidades transnacionales, se pueden rescatar factores que van mas allá de lo económico. Levit (ibid. 40) denomina a los mismos como "remesas sociales"; con ellas se refiere a ideas, conductas, identidades y capitales sociales que fluyen de un país a otro. Este punto de vista lo propone por primera vez Douglas Massey, quien presenta su teoría del capital social. Teóricos muy reconocidos, como Coleman y Pierre Bordieau, adoptan también esta terminología y siguen haciendo contribuciones en este área.

Podemos referirnos a las redes de migración como una forma de "capital social", ya que en ellas se desarrollan relaciones sociales que permiten el acceso a ciertos bienes, no sólo económicos sino también de otros tipos. Desde este punto de vista, las redes sociales se presentan como facilitadoras del proceso migratorio, sobretodo por las ventajas que proporciona al compartir información con sus miembros, al adquirir capacidad para enfrentar y superar los riesgos y los costos de la migración. 
Este enfoque también ha recibido críticas porque una de sus limitaciones radica en resaltar ese potencial que poseen las comunidades transnacionales para superar problemas, pero se dejan de lado aspectos negativos que también deberían considerarse en el amplio contexto de la globalización.

Al respecto, Canales, A. y Zlolniski reconocen que "las comunidades transnacionales no pueden concebirse únicamente en términos de redes de solidaridad y reciprocidad generalizadas que permiten resistir los efectos negativos de la globalización, sino que debe entenderse que su dinámica conlleva, al mismo tiempo, la reproducción de tensiones, conflictos y contradicciones que se dan en su seno y que, como tales, contribuyen a recrear el marco de desigualdad que condiciona la reproducción social de sus miembros" (op. cit. 414).

Las críticas a este enfoque nos permiten presenciar la complejidad de los elementos que intervienen en la conceptualización de la migración, y más si consideramos la diversidad de situaciones que caracterizan a los movimientos migratorios.

Tilly (citado por Canales y Zlolniski, 2002:421) manifiesta que la reproducción de las relaciones y estructuras comunitarias también adoptan una forma transnacionalizada en el sentido de que las estructuras culturales, sociales o políticas que constituyen cada comunidad también son transplantadas del país de origen a los lugares de asentamiento. Portes, Levitt y Basch ratifican esta situación cuando se refieren a la aparición de nuevos tipos de prácticas y símbolos como resultado de la interacción transnacional.

\subsection{Los sistemas migratorios}

Otro enfoque que considera la presencia de las redes como elemento explicativo importante en las migraciones es el enfoque de los sistemas, planteado por Akin Mabojunge en su estudio sobre la migración rural urbana en África. Desde esta perspectiva, se entiende como sistemas de migración a aquellos "espacios caracterizados por la asociación relativamente estable de una serie de paises receptores con un número determinado de regiones de origen" (Revista Internacional de Ciencias Sociales, 2000:42).

Se resaltará, sin embargo, que las asociaciones a las que aquí se hace referencia no son sólo el resultado de las corrientes migratorias sino que éstas se ven reforzadas por conexiones y vínculos de distinta naturaleza. Esta idea se explicita claramente en la definición que Moulier Boutang y Papademetriou presentan sobre sistema migratorio como "la combinación particular de tipos de flujos de población entre países de origen y países de destino con las leyes o reglas que rigen los fiujos y los organismos encargados de aplicarlas" (Domenachd y Picouet, 1995:104).

Los autores antes mencionados proponen una clasificación de seis tipos teóricos de los sistemas migratorios:

a) Asentamiento colonización.

b) Asentamiento inmigración.

c) Mano de obra instalación perdurable.

d) Mano de obra trabajadores "invitados".

e) Cierre País de retorno. 


\section{f) Cierre Minorías étnicas o país de inmigración.}

Se pueden identificar casos en los que algunos países mantienen estos tipos de sistemas simultáneamente en un momento determinado de su historia. A pesar de ello, se manifiesta que, de los sistemas antes mencionados, resultan tres principales: el de asentamiento, el de instalación perdurable y el de trabajo temporario. Los países receptores utilizarán éstos como fundamento para definir cuáles son sus políticas migratorias.

La crítica que se presenta a este enfoque es que apenas ha avanzado en la identificación de sistemas de migración internacionales y tal identificación se ha limitado hasta ahora a la parte más estable del sistema, que son los países situados en el extremo receptor.

Según Zlotnik (Revista Internacional de Ciencias Sociales, 2000:43), los elementos que definen la existencia de un sistema de migración son la homogeneidad estructural relativa, la contigüidad o proximidad geográficas, la similitud de políticas y la pertenencia común a organizaciones supranacionales.

Se hace referencia a un sistema, también en el caso de que un conjunto de elementos se reúnan para colaborar aunque sea indirectamente con los flujos migratorios. Las redes sociales que se entretejen alrededor de los procesos migratorios no están ausentes en este sentido, incluso en aquellos enfoques que resaltan que la migración responde a la demanda y oferta en el mercado de trabajo, porque reconocen que esto solamente puede ocurrir si se establece una red de instituciones que la facilite.

Entre los elementos que contribuyen al crecimiento de los flujos migratorios dentro de estas redes, Bob Sutcliffe menciona medios de transporte, información y contratación, modo de enviar dinero internacionalmente, comunidades en los países de origen en las que emigrar se considere aceptable, e incluso normal, y comunidades en los países de destino que faciliten la llegada de la persona migrante.

Con la migración, se van formando enlaces, redes y sistemas y, luego, su propia existencia tiende a perpetuar el flujo migratorio. Este argumento será sostenido por otro enfoque que resalta el carácter circular de la migración.

\subsection{Teoría de la causación acumulativa}

Desde el punto de vista de esta teoría, la emigración es un fenómeno que desarrolla su propia dinámica y que se perpetúa a sí mismo. Gunnar Myrdal propuso por primera vez este enfoque mediante la denominación de causación acumulativa, la misma que se manifiesta en un contexto de "efectos de repercusión" desencadenados por el desarrollo desigual en zonas subdesarrolladas.

Según esta lógica, cada vez, la migración tiende a causar más migración, en un círculo de causación acumulativa, así que, aquellas comunidades que se han incorporado a un sistema migratorio internacional manifestarán una fuerte tendencia a seguir en él. Sólo el surgimiento de nuevas circunstancias de importancia que lo impidan provocarán la ruptura de este círculo.

Actualmente, Douglas Massey amplía este concepto cuando identifica una serie de factores y mecanismos que dan lugar a que la migración se perpetúe. 
Este autor manifiesta que la migración modifica la realidad de tal forma que induce a desplazamientos subsiguientes por medio de una serie de procesos socio-económicos. Uno de esos procesos, y al que se le atribuye mayor importancia, es el de la expansión de las redes, al que se suman otros mecanismos que son generados por la migración y, al mismo tiempo, contribuyen a que ésta se produzca: la privación relativa, el desarrollo de una cultura de la migración, una distribución perversa del capital humano y la estigmatización de los trabajos que suelen realizar los inmigrantes.

En este enfoque teórico, se considera las redes como un fenómeno que se reproduce a sí mismo y permite que la migración se auto-alimente independientemente de otros condicionamientos. Froud (citado por Martínez Veiga, 1997:132) manifiesta que en este proceso "el primer paso es el único dificil, dado que, una vez que se ha establecido a gran escala, la emigración se alimenta a si misma. Una vez que se han asentado, se multiplican y atraen tras de si a sus parientes".

Ubaldo Martínez Veiga denomina a este primer paso el "momento de movilización" que estará impulsado por factores no de carácter individual sino de carácter colectivo. Manifiesta que el proceso migratorio está engastado socialmente dentro de una red de relaciones. Esta red no se creará en el proceso migratorio, sino que ya existía con anterioridad a él, sólo que ahora será activada, pues antes estaba en estado latente. Desde este punto de vista, las relaciones de parentesco, principalmente, así como las de amistad y las establecidas en un origen común contribuirán junto con los elementos antes mencionados a que el círculo de la migración no se rompa.

\section{EL CONTEXTO MIGRATORIO NACIONAL Y LAS REDES FA- MILIARES}

Se menciona continuamente que, si bien la migración internacional se ha acentuado en los últimos tiempos, ha sido constitutiva del desarrollo histórico de la humanidad. Actualmente, las explicaciones de este tipo de migración deben enmarcarse en el contexto de un mundo globalizado, incluyendo elementos que hagan referencia no sólo a los factores económicos, sino también a los políticos, culturales, sociales, etc. Los últimos estudios ${ }^{3}$ señalan la necesidad de incluir también los factores individuales, psicológicos y emocionales.

En el caso de Ecuador, la crisis económica y política ocurrida en el período 1997/2000 -por factores como el Fenómeno del Niño, la crisis financiera internacional, el peso de la Deuda Externa y la caída de los precios de productos de exportación, el mal manejo de la política económica por parte del Gobierno, el feriado bancario, etc- permite presenciar un gran cambio con respecto a los flujos migratorios. Estos factores de tipo económico y político presentarán sus repercusiones en el área social y también psicológica.

La lógica que caracterizaba el proceso migratorio de los ecuatorianos a otros países se transforma a partir de la acentuación de la crisis. Uno de esos

3 Cartillas sobre Migración, Plan Migración, Comunicación y Desarrollo. 
cambios se manifiesta en la elección masiva de un nuevo país de destino: España. Según la Dirección Nacional de Migración, 504.203 ecuatorianos -más del $10 \%$ de la población económicamente activa- salieron del país entre 1999 y 2000. Antes de 1995, el principal destino de los migrantes era los Estados Unidos, de ahí en adelante se han incrementado los flujos migratorios a España. Según la "Encuesta de medición de indicadores sobre la niñez y los hogares" (EMEDINHO), levantada en noviembre de 2000 por el INEC, el $65 \%$ de los ecuatorianos que salieron en busca de trabajo antes de 1995 se dirigieron a Estados Unidos, desde ese año en adelante el 53\% de los migrantes fueron a España.

Son diversas las explicaciones que se pueden dar a este cambio de tendencias, como "la eliminación de requerimientos de visa de entrada, el idioma, las redes familiares y de transporte de migrantes que se han armado en los últimos años" (SIISE, 2001).

Cuando se hace mención a los factores psicológicos de la migración, se analiza como ésta "pasa de ser una estrategia individual para convertirse en una estrategia general de subsistencia, ya no sólo del individuo sino de las familias" (Plan Migración, Comunicación y Desarrollo, 2003: 8). De ahí que se reconocerá a éste como un fenómeno social que se constituye en un instrumento de supervivencia y reconocimiento social.

De esta forma, la migración comienza a ser vista como una estrategia familiar, en la cual las redes sociales contribuirán a consolidar este proceso. Otro de los factores de tipo psicológico, a los que se hace referencia continuamente, es aquél en el que la motivación migratoria responde a las conductas de imitación a los que ya se fueron. Este hecho ha llevado al surgimiento de términos que califican a tales actitudes como una especie de "contagio social", en el sentido de que actúan, como motivaciones de la migración, la necesidad de reagrupar la familia, por ejemplo, así como la información emitida por los emigrantes, que tiene una gran carga simbólica.

Alba Goycoechea Rodríguez (2003:9) manifiesta que, además de las condiciones materiales que son externas y no controlables por los individuos, existen otros factores, los subjetivos, que se construyen a partir de las aspiraciones, expectativas y valoraciones de los involucrados en este proceso. Serán estos factores subjetivos los que se van a manifestar fuertemente en las redes migratorias.

Goycoechea considera que se deben tomar en consideración estos factores que intervienen fuertemente en las redes sociales, con la finalidad de poder entender aspectos sociales comúnmente no contemplados, como el carácter de selección de los migrantes, la selección de los destinos, la canalización de los recursos, la calidad de vida, la persistencia de los flujos migratorios. De ahí que debamos analizar las redes familiares como elementos que faciliten la explicación de los flujos migratorios. 


\section{IMPORTANCIA DE LAS REDES SOCIALES EN EL PROCESO MIGRATORIO}

Es esencial abordar el estudio de las redes sociales como uno de los factores explicativos de la migración. Frecuentemente, se escucha que, en los países con una cultura de emigración, muchos migrantes se desplazan porque tienen familiares en el país de destino, pues esas redes tienen un efecto multiplicador que es evidente.

Las redes han cobrado un gran realce, especialmente ahora que la migración a ciertos países está muy restringida. Esta situación tiene su explicación básicamente en dos factores: primero porque en muchos países la reunificación familiar constituye una parte considerable de las corrientes de inmigración y, por otro, porque la importancia de las redes sociales está abocada a aumentar a medida que la entrada en los países receptores sea más difícil, por su capacidad de reducir los costos y riesgos del desplazamiento (Revista Internacional de Ciencias Sociales, 2000:42).

Cuando se analiza la importancia de las redes, se menciona que son el principal mecanismo que hace de la migración un fenómeno que se perpetúa, su naturaleza es acumulativa, con tendencia a crecer, facilita los desplazamientos ulteriores que a su vez amplían las redes y la posibilidad de expandirse en el futuro.

Esta importancia de las redes se manifiesta en aquel enfoque explicativo según el cual la migración puede continuar con independencia de las causas que llevaron al desplazamiento inicial, por lo que son con frecuencia los mejores indicadores de flujos migratorios. Faist resalta la importancia de las redes porque constituyen un nivel de relación intermedio entre el plano micro de la adopción de decisiones individuales y el plano macro de los factores determinantes estructurales.

Otro elemento que interviene en este análisis es el gran valor cultural, ideológico, que los migrantes atribuyen a las redes de familiares y de amigos, porque les permiten despojarse en alguna medida de los temores y tensiones que representan los riesgos y los costos de la migración. En este ámbito, influye también la imagen positiva del país elegido como lugar de destino, generado por la información que trasmite la red. Esta información en algunas situaciones llevará a sobrevalorar las condiciones de desarrollo y de modernidad del país de destino.

Los elementos antes mencionados dejan ver a las redes como dinamizadoras del proceso migratorio, que involucra no sólo a los individuos sino a las familias como verdaderas "unidades migratorias", cuando despliegan una serie de estrategias que gestionan este proceso. De ahí que la migración esté inmersa en un conjunto de vínculos que advierten la presencia de redes sociales. 


\section{CRECIMIENTO O PERSISTENCIA DE LOS FLUJOS MIGRA- TORIOS A TRAVÉS DE LAS REDES SOCIALES}

Si mantenemos la afirmación de que las redes sociales, $y$, especialmente, las redes familiares en el caso ecuatoriano, cumplen una función importante dentro de la migración, para ratificar tal afirmación se debe recurrir a algunos datos que manifiesten la presencia de ellas y su función como canalizadoras de este proceso. En una investigación desarrollada en Murcia, los resultados dan a conocer el predominio de las redes familiares. A la pregunta: ¿con quién vino a España?, la población investigada responde en un $75 \%$ con familiares y en un $25 \%$ con amigos. También se les pregunta: cuando llegó, ¿quién le recibió?. El $84 \%$ manifiesta haber sido recibido por familiares, el $6 \%$ por amigos y el $12 \%$ por nadie (Botero, 2000:42).

No hay duda de que la presencia de redes familiares es muy fuerte. Luego se ubican las redes de amigos, que serán sobre todo aquéllas que funcionan en un inicio, ayudando a los ecuatorianos a insertarse en el medio español, quienes luego apoyarán también a que sus familiares emigren. Ese es el punto de partida que conforme al paso del tiempo dará lugar al incremento de la emigración.

En el mismo estudio, se realiza un análisis de cómo se van constituyendo estas redes desde un inicio: "a mediados de la década de los 90 llegaron a Europa aquellos que, sabiéndolo o no, comenzaron a colocar los hilos de una red que se irá extendiendo poco a poco hasta llegar a conformar un complejo y rico entramado. Hermanos, tíos, primos, amigos, sobrinos y una gran variedad de afinidades recién descubiertas funcionaron para que el paisaje social de países como España comenzara a cambiar" (ibid, 6).

Los relatos de los familiares y de los propios emigrantes demuestran que fue en esta época cuando los primeros ecuatorianos empiezan a establecerse en los diversos territorios de España. La migración más antigua se ubica en 1995 y la más reciente en agosto de 2003, justo antes de que se aplique el visado, pero existe una media de entre 4 y 6 años de migración que es la que predomina.

"Mis cuñados se fueron primero y luego le llevaron a mi esposo diciendo que ha sido bueno el trabajo. Unos españoles vinieron, les dijeron a mis cuñados vamos a darles la visa para llevarles allá, así se fueron algunos de mis cuñados hace 8 ó 9 años, los otros están 5 años, 4 años y 3 años". Exmigrante ecuatoriana en Loja, Ecuador.

En algunos casos, de acuerdo a los relatos, fue posible identificar a los llamados "pioneros", quienes se establecieron en el lugar de destino y comenzaron a contribuir en el incremento de los flujos migratorios, especialmente de los familiares.

"El primero que ha llegado ha sido mi primo Segundo Yupanqui, ellos han sido los guiadores. Él vive en San Javier, no sé cuánto tiempo llevan aquí, ya se van dos veces para el Ecuador, por alli unos 7, 8 años. Son los primeros que salieron del Cañar. Antes era poquita la gente, 
cuando alabábamos al Señor éramos apenas 6 personas, luego llegaron los primos de Ecuador y ahora somos 60".

Emigrante ecuatoriana en SAN JaVier, ESPAÑa.

Es evidente que las redes familiares se presentan como uno de los elementos que inevitablemente contribuyen al incremento de los flujos migratorios desde el lugar de origen. En muchos casos se identificaron familias de hasta 7 integrantes que han emigrado por medio de la ayuda brindada por estas redes.

"Todos los hijos de mi abuelita están en España, son 6, después de mi mami se fue otra tía que se casó y se fue".

HiJA DE EMIGRANTE EN LOJA, ECUADOR.

Las redes tienen la capacidad de multiplicarse porque cada vez se van incorporando nuevas personas. A partir de la aprobación de la visa como requisito para ingresar en territorio español, el efecto multiplicador de las redes se ha visto afectado. De acuerdo a los datos proporcionados por la Embajada de Ecuador en España, la llegada de ecuatorianos se ha reducido en un $80 \%$. Estos datos dan a conocer que la emigración no ha cesado, que sigue desarrollándose a pesar de las restricciones establecidas.

\section{PAPEL DE LAS REDES EN EL PROCESO MIGRATORIO}

Si partimos de la idea de que muchos migrantes efectivizan la posibilidad de desplazarse porque otros con los que están relacionados han emigrado con anterioridad, podemos considerar importante el papel que desempeña la red en el proceso. Ubaldo Martínez Veiga señala que son tres los elementos que aumentan fuertemente la probabilidad de la emigración:

1. La experiencia migratoria previa del propio individuo.

2. La presencia dentro de la misma unidad doméstica de otro miembro de la familia que haya migrado.

3. La residencia en un pueblo o comunidad donde haya tasas de emigración alta.

Tomando como referencia estas pautas, se analizará qué sucede con el caso de la emigración de ecuatorianos a España.

\subsection{La experiencia previa del propio individuo indudablemente facilita la emigración}

A este factor se pueden sumar otros como la desilusión de aquéllos que retornan frente a la mala situación económica del país.

"Mis hijas regresaron dos veces a Ecuador, la primera vez para quedarse pero vieron que la situación del país tampoco ofrece muchas posibilidades, intentaron encontrar trabajo y se encontraron con los sueldos bajos". Padre de emigrantes en Cañar, Ecuador.

La posibilidad de emigrar nuevamente estaba latente, ya que tenían la experiencia de migración reciente. Otros factores también importantes para los migrantes incrementarán considerablemente los flujos migratorios. En algunos 
casos, la emigración es vista como la posibilidad de escapar ante situaciones dolorosas que se vuelven insoportables.

"La primera parte del sufrimiento ya la has pasado, y te vuelves. Al menos, el panorama que yo te cuento de mi madre enferma y luego le encuentras a la persona que tú quieres con otra persona, tú quieres escapar de ello, salir nuevamente de alli, no puedes aguantar el dolor... entonces eso te hace pensar que tienes que volver porque alli ya no tienes nada, aunque tienes tu familia".

Emigrante ecuatoriana en Madrid, España.

"Él no se iba a ir allá prácticamente, para evitar todo esto él se fue, ya no le dejaban en paz, le lacraron la cara, una vez cogieron la botella y con eso le dieron. Todos le apoyamos, no podiamos aguantar a los jóvenes todo el día amenazando".

Hermana de un emigrante en Cuenca, Ecuador.

Los factores subjetivos no pueden dejarse de lado en el momento de analizar las principales motivaciones que llevan a los ecuatorianos a emigrar. Se debe reconocer que el aspecto económico es una de las motivaciones fuertes, sin embargo, ante la necesidad de superar conflictos en el ámbito familiar o social, la migración será vista como la salida a dichos conflictos.

\subsection{La presencia dentro de una misma unidad doméstica de otro miembro de la familia que haya emigrado}

De los casos estudiados, se puede presenciar un predominio de las redes familiares que se convierten en uno de los elementos de atracción y estructuración del proceso migratorio. Entre los familiares, predominan fundamentalmente los hermanos, luego, se ubican los primos y, en algunos casos, es el esposo o esposa quien llama a su pareja. Cuando un miembro de la familia llega al país de destino y se establece, podrá ayudar a sus familiares a cumplir el mismo proyecto.

Encontramos casos de familias muy amplias, generadas por la "migración en cadena", que se empiezan a ampliar más todavía cuando sus miembros establecen relaciones sentimentales con personas del país de destino y luego se hacen presentes los hijos.

"Ruth está enamorada, cuenta que tiene un novio español, creo que se quiere quedar allá". MADRE de una EMIGRANTE ECUATORIANA EN CAÑAR, ECUADOR.

"Mi cuarta tía se casó con un español".

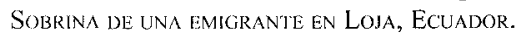

\subsection{La residencia en un pueblo o comunidad en donde haya tasas de emigra- ción alta}

Son pocos los casos de ecuatorianos que viajan con ayuda de sus amigos, sin embargo, se puede observar que los mecanismos de información desarrollados por estas redes son útiles principalmente para la preparación del viaje así como para la llegada. Este tipo de ayuda no tiene grandes diferencias con respecto al de las redes familiares, ya que estarán presentes en todo el proceso, 
incluida la acogida en España y la colaboración que requieren para insertarse a la nueva situación que no se reduce al ámbito de lo laboral.

En el caso de que los amigos no sean los que contribuyan en el desarrollo del proyecto migratorio, se acuden a otras estrategias, como son las Agencias de Viajes, que son vistas como un medio confiable. Si unos de los miembros de la familia viajó inicialmente por este medio, se puede ver que los próximos familiares que pretendan emigrar utilizarán también esta estrategia, a pesar de contar con el apoyo del familiar que ya se encuentra en España.

"Para venir, fui a la Agencia «Ecuaturis» de Cuenca, mis primos eran clientes de la agencia". Emigrante ecuatoriano en San Javier, España.

A este elemento responde también la concentración de ecuatorianos de determinadas regiones en lugares de destino comunes. Así, "encontramos en Barcelona emigrantes que proceden de la costa, en Madrid se ubican los de la capital ecuatoriana, en ciudades pequeñas, como Murcia o Alicante, los cuencanos y lojanos y en lugares pequeños, como en Balsicas, Pilar de la Horadada, Jumilla, San Javier o San Cayetano, campesinos e indigenas " (citado en Revista Trimestral del Centro Studi Emigrazione, Roma, 2.004:351).

'Llegué a Jumilla donde mis amigos, es una localidad de Murcia, llegué desde el primer dia y no he salido desde ese momento, ahora voy para mi tercera tarjeta de trabajo". Emigrante ecuatoriano en Jumilla, España.

"Ya tenian donde llegar por eso toditos están ahí (Pilar de la Horadada). De ahí dicen que en otras partes hay más trabajo, dicen que ganan más pero no sé, ya no salen a ningún lado. Dice que ahi está bastante gente de San Agustín". Familiar de emigrantes de Cuenca, Ecuador.

Alejandro Lorca manifiesta que la formación de este tipo de redes sociales es lo que explica las especializaciones de la inmigración en función del territorio y del origen cultural (Lorca y otros, 1997: 30 35).

A estos elementos, debemos sumar los que Martínez Veiga denomina como "cultura de la emigración". Se habla de una cultura porque el fenómeno migratorio es tan frecuente que siempre se presenta como una posibilidad que está a la mano y a la que se puede recurrir constantemente, también se implica con este término la idea de que la migración ha calado fuertemente dentro del mundo simbólico en estos países.

\section{FUNCIONES DE LAS REDES}

Dentro de las funciones que cumplen las redes, podemos mencionar lo que Martínez Veiga denomina "las relaciones de vuelta" hacia el país de origen, en donde ubica primordialmente el envío de remesas y la frecuencia del contacto.

Con respecto al envío de remesas, casi la totalidad de emigrantes envían remesas al Ecuador, que van desde los 100 hasta los 600 u 800 dólares. Hay pocos casos en los que se envían cantidades superiores a los 1.000 dólares, las mismas que son destinadas fundamentalmente al ahorro; esto sucede cuando es una pareja la que trabaja, una vez que ya ha pagado la deuda. 
Cuando el monto es de 100, 200 ó 300 dólares, las remesas están destinadas a la manutención de los hijos y a su educación. Los que envían 500, 600 u 800 dólares destinan las remesas además de a la manutención y educación de los hijos a pagar sus deudas o a terminar la construcción de la casa que han iniciado.

Con respecto a la frecuencia del contacto, debemos considerar el envío de las remesas, que en casi todos los casos es mensual, incluso cuando los hijos solteros envían remesas a sus padres. En cuanto a la frecuencia de la comunicación, las madres y luego los padres son los que con mayor frecuencia se comunican con sus hijos, en algunos casos lo hacen cada semana, otros cada 15 días.

Es necesario considerar también las expectativas y posibilidades de los miembros de la red de reagrupar su familia, con la finalidad de estimar su sentido de permanencia. Cuando los y las emigrantes hablan de la reunificación familiar piensan primordialmente en sus hijos. Hay pocos casos de ecuatorianos que ya han realizado los trámites y han reunificado su familia. Un grupo mayoritario señala la necesidad de tener cerca a sus hijos, pero no han realizado ninguna gestión al respecto y tampoco tienen planificado hacerlo. Afirman que no es fácil el trámite a seguir. Aquéllos que tienen claro que su estancia en España es temporal no piensan en aquella posibilidad.

También es posible identificar las funciones que cumplen las redes en el pais de destino. Gurak y Cases (1998) señalan que estas funciones se pueden clasificar en dos amplias categorías: primera, la función de adaptación y selección. La adaptación se refiere a la "acomodación a corto plazo" a duras condiciones temporarias y a la integración en las instituciones principales de la sociedad de destino a largo plazo. También la red ejercerá influencia en la selección de quién y cuándo migra. Segunda: la función de canalización, ya que la red cumple un gran papel a la hora de determinar los principales lugares de destino de los migrantes. Los primeros migrantes son los primeros que ayudan a establecer grupos específicos al identificar lugares posibles de destino.

En cuanto al papel que cumple la red en la canalización a la hora de determinar el lugar de destino, la mayor parte de los emigrantes ecuatorianos viajan con la ayuda de sus familiares y eligen como lugar de llegada aquél donde se encuentran ellos. Para muchos, el lugar de llegada se convierte en el lugar de asentamiento. Esto no quiere decir que con el paso del tiempo no se contemplen otras posibilidades.

Con respecto a la función de adaptación, la red es fundamental en el momento de llegada, hasta el establecimiento y hasta que los emigrantes logren insertarse en el mercado laboral. Queda demostrada tal afirmación cuando se ve que quienes optan por el cambio tienen que pasar un tiempo considerable en el lugar de llegada. En etapas posteriores, la red ya no juega el mismo papel, su importancia será menor, en algunos casos el cambio se realiza incluso sin tener familiares en el nuevo lugar seleccionado.

El papel de la red es valorado en el momento de la llegada cuando les acogen en sus viviendas, pero se resalta más el papel de la red cuando se habla del mercado laboral. Los migrantes señalan que fueron sus familiares quienes 
"les dieron consiguiendo trabajo", en otros casos, por lo menos les proporcionaron información. Hay que resaltar la importancia de la red, ya que muchos llegan directamente a trabajar gracias a la ayuda de sus familiares y amigos. Sin embargo, hasta tanto, sin la presencia de estos mecanismos de solidaridad, la inserción a este nuevo espacio sería más dura.

No hay duda de que, en esta etapa, el papel de la red se centra más en la prestación de asistencia a la adaptación a corto plazo con el apoyo brindado mediante la vivienda, el dinero, la información, el trabajo y las estrategias generales de supervivencia.

No es notoria la presencia de las redes en la adaptación a largo plazo, sobre todo para facilitar la integración con las instituciones principales en la sociedad de destino. Sin embargo, la información sigue siendo un canal muy importante, sobre todo cuando los emigrantes ven la necesidad de regularizar su situación y sus familiares y amigos ya han realizado dichos trámites. La experiencia propia les lleva a informar sobre el procedimiento a seguir y sobre las instituciones a las que pueden acudir para tal fin.

Como se señaló anteriormente, la red de familiares es la predominante en el caso ecuatoriano, por eso, en el momento de seleccionar quiénes deben migrar, se considera a los familiares más cercanos para que lleven a cabo el proyecto migratorio.

\section{TIPOS DE REDES SOCIALES}

De las redes sociales, se presentan diferentes tipos de clasificaciones. De acuerdo a su origen, se clasifican en redes emisoras, que son aquellas conexiones que existen en el lugar de origen, y redes receptoras, que son las que se establecen en el lugar de destino. Según Martínez Veiga, la separación entre las redes de origen y de destino tiende a difuminarse. De acuerdo a las teorías antes mencionadas, aquellas redes que juntan el lugar de origen y de destino darán lugar a la formación de las comunidades transnacionales.

En los países de origen, como en los de destino, se establecen redes sociales y familiares y, a través de ellas, los miembros de las comunidades transnacionales realizan sus actividades sociales, económicas, culturales, etc. La transnacionalización familiar tiene múltiples manifestaciones en el caso de los ecuatorianos. Ésta se presenta cuando en el núcleo familiar, al no poseer recursos suficientes para la emigración simultánea, las familias deciden enviar primero a uno de sus miembros.

Esta primera etapa del ciclo migratorio apunta a la obtención de los recursos que posibiliten una posterior reunificación familiar.

Con respecto a la financiación del viaje, la forma más común en Ecuador para conseguir el dinero es a través de los "chulqueros"4. Aquí, juega un papel muy importante la ayuda de la familia, ya sea para establecer el contacto con el prestamista porque tiene relaciones de amistad con él o para conseguir el préstamo sin necesidad de hipotecar casas o terrenos.

4 Personas que prestan dinero de forma ilegal para financiar el viaje al extranjero a cambio de altos intereses. 
Muy pocas personas acuden a préstamos bancarios, por las grandes exigencias que ello implica; por eso, a pesar de que acudir a los chulqueros significa pagar intereses desde el $4 \%$ hasta el 10 ó 12\%, consideran que éste es uno de los medios que facilita la puesta en marcha del proyecto migratorio.

"Ahora una amiga trabaja prestando dinero, bueno pero veo que eso es justo porque te salva, con los chulqueros hay facilidades, en el banco en cambio, te ponen demasiadas trabas".

Emigrante ecuatoriano en Barcelona, España.

Cuando el familiar que está en el país de destino ha pagado ya su deuda, pero no ha logrado ahorrar lo suficiente como para financiar el viaje de su familiar, ofrecerá esos pequeños ahorros para que la deuda con el chulquero no ascienda demasiado.

El monto que se pide a los chulqueros va desde los 3.000 hasta los 6.000 ó 7.000 dólares, de los cuales 1.500 ó 2.000 son devueltos en la mayoría de los casos, ya que se los considera como "la bolsa de viaje", éste era un requisito que debía ser presentado en el país de destino porque los emigrantes ingresaban supuestamente como turistas.

Los casos más llamativos en donde intervienen las redes para facilitar el viaje de los familiares son aquéllos que se dan fundamentalmente entre esposos y hermanos.

En el caso de los esposos, uno de ellos, generalmente el hombre, es el que primero emprende el proyecto migratorio. Luego de establecerse en el país de destino y salvar la deuda, el migrante comienza a ahorrar para contribuir con el financiamiento del viaje de su esposa. Hay que tomar en consideración que no siempre se tenía planificado el viaje desde un inicio, pero con el pasar del tiempo surgen este tipo de proyectos incentivados por diferentes factores.

"Mi esposo me mandó el dinero desde España, yo me fui así no más a visitarle, él estaba año y tres meses allá cuando yo me fui".

ExMigrante EN LOJA, ECUADOR.

El tiempo de pago de la deuda, generalmente, va desde los seis meses a un año, en algunos casos menos. Este tiempo se ve reducido considerablemente cuando se destinan los recursos económicos de la pareja para este fin. En el caso de los hermanos, el tiempo de migración entre ellos es un poco mayor: de dos años en adelante.

"Mi hermano viajó hace cinco años y mi hermana viajó hace un año y tres meses, se endeudó con mi hermano mismo pero ya le acabó de pagar la deuda: ahora están con el proyecto de hacerle una casa para mi mami". Hermana de emigrantes en Cuenca, Ecuador.

Las explicaciones que giran en torno a los factores por las cuales los emigrantes apoyan a sus familiares para que migren también pueden ser varias, sin embargo, los testimonios obtenidos en la investigación nos permiten afirmar que el miedo a la soledad es uno de los factores importantes que se presencia en algunos casos, tanto entre las parejas como entre los hermanos. 
"Los que estamos en pareja estamos bien, a veces pasar solos los hombres es duro. Los que pasan sin mujeres, sin hijos pasan mal, otros que vienen sin familia se olvidan de la mujer, de los hijos, de la deuda". Emicrante de Cañar en San Javier, España.

"Primero se fue la hermana y luego él (hermano) le daba pena, se llevaban muy bien los dositos, son seguiditos, entonces dijo: yo me voy papi, ayzideme que me voy, no hubo poder humano que lo detenga y ya está allá". Madre de emigrantes en Madrid, España.

Los testimonios antes mencionados dan a conocer que son los factores subjetivos lo que impulsan a los familiares a efectivizar el proyecto migratorio, ya sea por temor a la soledad, por la pena que provoca el familiar que emigra o por la necesidad de afecto en el caso de las parejas.

\section{DIMENSIONES INSTRUMENTALES DE LAS REDES}

Los antropólogos de Manchester manifiestan que, además de la dimensión comunicativa de la red, hay una dimensión instrumental porque las redes migratorias sirven para obtener ciertos resultados. En este caso, podemos mencionar la posibilidad que ofrecen las redes para encontrar trabajo en el país de destino como una de las dimensiones con mayor peso para los migrantes.

Las relaciones transnacionales iniciadas por el intercambio de bienes de diferente naturaleza entre el país de origen y el de destino se manifiestan en la ayuda que pueden brindar las redes sociales en la búsqueda de trabajo y la inserción laboral, que no sólo se reduce a las opciones laborales disponibles en los lugares de asentamiento de los migrantes sino que también incluye la demanda desde los países de origen. Esto es posible gracias a la información difundida en doble dirección sobre la necesidad y las opciones de trabajo, en donde la estructura de las redes sociales $\mathrm{y}$, especialmente, familiares desempeñan un papel sumamente importante.

A pesar de que no resulta sencillo la búsqueda de fuentes de trabajo en el país de destino, los emigrantes que recién llegan cuentan con el apoyo de sus familiares. Si las gestiones para conseguir el empleo que se requiere para el nuevo emigrante no se han realizado con anterioridad al viaje, éstas se iniciarán cuando él llegue. En este caso, se hará uso de las relaciones, ya sean laborales o de amistad que se han podido establecer en el tiempo de permanencia en España.

"Mi suegro hablaba con los jefes y les decia que le den faena (trabajo) para su yerno que está parado y ellos le preguntaban quién es, cuántos años tiene, le averiguaban todo y luego le decian que me lleve".

Emigirante ecuatoriano en Jumilla, España.

Los ecuatorianos, inicialmente, no tienen la posibilidad de elegir el trabajo, más aun si se encuentran en situación irregular. Por tal razón, toda oferta será válida, ya que las necesidades en ese momento son urgentes. Por un lado, el emigrante que recién llega tiene que conseguir trabajo con el fin de obtener recursos para el mantenimiento tanto de él en España como de la familia en 
Ecuador, también se debe iniciar el pago de la deuda adquirida en el país de origen generalmente.

Los emigrantes que recién llegan encuentran mayores posibilidades de conseguir trabajo en el campo para el desarrollo de actividades agrícolas. En el caso de las mujeres, las oportunidades laborales son mayores porque al trabajo en el campo se suma la demanda de este género en el área del servicio doméstico en las casas o el cuidado de niños y ancianos.

\section{MOVILIDAD SOCIAL EN EL ÁMBITO DE LAS REDES}

En el análisis que se realiza de las redes sociales, se consideran no sólo los factores positivos que en ellas se desarrollan sino también factores nada favorables para sus miembros. En este sentido, las redes pueden impedir la movilidad social debido a las relaciones estrictamente cerradas que en ellas se establecen. Se identificaron casos de emigrantes que, luego de haber transcurrido un tiempo considerable en un lugar de trabajo conseguido por un familiar o amigo, empiezan a mirar una serie de alternativas que se presentan en el medio. Obviamente, para que esto suceda, las condiciones iniciales de irregularidad, de falta de conocimiento del país de destino han cambiando. De ahí, podemos plantear que, en las redes, la movilidad social sí es posible, así tenga que transcurrir un tiempo para que ello suceda.

De acuerdo a los datos obtenidos, esta visión de las alternativas posibles por parte de los emigrantes surge complementada con la seguridad que proporciona el haber logrado regularizar su situación.

"Cuando ya tienes tus papeles todo cambia y dices: ¿por qué me voy a aguantar aqui? Ya no quiero trabajar de interna en esta casa, quiero trabajar de externa. Me quedé trabajando y cuando ya trabajas de externa sales más, conoces más gente, ves más mundo en el buen sentido". Emigrante ecuatoriana en Madrid, España.

"Cuando ya tenía mi documentación cambió mi situación, después de la obra (construcción), ahora estoy en una empresa de mallaje, esta empresa pone mallas en las autopistas, autovias. Y con la documentación uno ya podia hablar duro con los jefes, cuando no tenía me quedaba callado, echaba la vista abajo".

Emigrante ecuatoriano en Jumilla, España.

Otro aspecto que demuestra que, en las redes, si se presenta la posibilidad de movilidad social para mejorar sus condiciones, se manifiesta, en algunos casos, cuando el familiar que emigró primero cede su puesto de trabajo al familiar o amigo que recién llega, porque él ha podido conseguir otro.

"Yo tenía una amiga en Barcelona que me ofreció prestarme el dinero para el viaje y también me ayudó a conseguir empleo. Luego yo le traje a mi hermana y le dejé en mi trabajo".

Emigrante ecuatoriana en Barcelona, España. 
Se puede afirmar entonces con seguridad que, al existir círculos cerrados de relaciones en las redes sociales, se retrasa la movilidad social, pero no se la impide.

Tampoco es posible desconocer aquellos factores generados por las propias redes para reducir el ámbito de relaciones sociales con otros integrantes que no formen parte de las mismas. Esto se puede constatar con los testimonios de emigrantes que mantienen relaciones sólo con sus compañeras/os de trabajo, con su familia o con paisanos.

"No tengo amigos, contadas una o dos amigas que son del barrio de uno mismo (Loja). Como he estado de interna no he tenido la oportunidad de hacer amigos, aqui mis hermanas son mis amigas, converso con ellas, nos dedicamos al trabajo, no salimos, no hay tiempo de hacer amigos". Emigrante ecuatoriana en Barcelona, España.

Los familiares de los emigrantes en Ecuador también manifiestan que las relaciones que se establecen en España son básicamente con paisanos.

"Allá se relacionan más con ecuatorianas, tienen amigas que son qui-

teñas". Familiar de emigrantes en Cañar, Ecuador.

Los factores que contribuyen a explicar este tipo de situaciones residen en la falta de tiempo para establecer nuevas relaciones, tiempo que está designado principalmente al trabajo y al descanso cuando es posible. Otro factor, que, necesariamente, coincidiría con aquellos enfoques que sostienen que las redes, al formar círculos cerrados, impiden la movilidad social, es aquel ambiente de bienestar que proporcionan las redes familiares, sobretodo ambiente que reproduce prácticas religiosas y culturales, en algunos casos, de tal forma, que no se mira más allá de esas relaciones establecidas en el círculo de la red.

\section{CONCLUSIONES}

La incorporación de los factores sociales en el análisis de la migración permite considerar las redes como un elemento constitutivo de este proceso, a partir de una diversidad de enfoques teóricos como el de las comunidades transnacionales, los teoría de los sistemas y la de la causación acumulativa, mencionadas en este artículo de forma general.

En el caso de la migración de los ecuatorianos a España, resulta de gran utilidad la inclusión de las redes en el análisis de este proceso. Así, se presencia un predominio de las redes familiares sin que ello excluya a las redes de amigos y de instituciones, organizaciones y asociaciones que se han establecido en el país de destino.

Las redes familiares cumplen diversas funciones tanto en el país de origen como en el de destino, las mismas que facilitarán en muchos casos la puesta en marcha del proyecto migratorio. A partir de 1995, se empiezan a establecer en España los primeros emigrantes ecuatorianos que sentarán las bases de la constitución de extensas redes con el pasar del tiempo. Este hecho ayuda a explicar el incremento de los flujos migratorios. 
Las funciones que desempeñan las redes en el país de destino adoptan una importancia fundamental, sobre todo en el momento de llegada. La concentración de emigrantes de determinadas zonas de Ecuador en lugares específicos de España se puede explicar a partir del papel que cumple la red en la selección de dichos lugares, en los cuales se han establecido los primeros emigrantes ecuatorianos.

Otra de las funciones de gran importancia en el momento de llegada de los emigrantes es la presencia de relaciones de colaboración y ayuda mutua que son las que predominan especialmente en el momento de la inserción en el mercado laboral. Las dimensiones instrumentales de la red se destacan en el momento de la inserción de los nuevos emigrantes en el medio español.

Una limitación que viene de la mano de la asistencia que prestan las redes a sus miembros es la reducida gama de posibilidades de salir del círculo creado por ellas. Si a esto se suma la situación de irregularidad de los emigrantes, la búsqueda de otras oportunidades en el campo laboral estará impedida por la inseguridad que ello genera.

\section{BIBLIOGRAFÍA}

ASTUDILLO José, "La emancipación del sujeto como supuesto básico, para convertirse en actor(a), y construir redes sociales alternativas", Investigación previa a la obtención del título en Master, Cuenca, 2003

BOTERO Luis Fernando, "Se me borró la cinta" (Economía, subalternidad y cultura. El caso de los ecuatorianos en Murcia), Ediciones Abya Yala, Quito Ecuador, 2000

CANALES Alejandro y ZLOLNISKI Christian, "Comunidades de Migrantes", en Simposio sobre migración internacional de las Américas, San José, Costa Rica, Septiembre del 2000

Cartillas Sobre Migración, "Las remesas de los emigrantes y sus efectos en la economía ecuatoriana", N. 1, Mayo del 2002

CASTELLS Manuel, "La era de la información" (La sociedad red), Vol, 1, Alianza Editorial, Madrid, 2000

CASTILLO Manuel Ángel, "Migración Internacional en las Américas", (Las consecuencias sociales de la Migración en el hemisferio y sus relaciones con las políticas sociales), Santiago de Chile, 2002

CEMLA, "Estudios Migratorios Latinoamericanos", Argentina, Revista N. 49, Diciembre del 2001

Colectivo IOÉ, "Ecuatorianos en Barcelona, Línea de Investigación N. 6, Madrid, 2002

Congreso de Estudiantes de Trabajo Social, “Migraciones y Redes Sociales”, Ediciones Universidad de Salamanca, España, 2002

DE LUCAS Javier y TORRES Francisco, “Inmigrantes: ¿cómo los tenemos? (Algunos desafíos y (malas) respuestas), Ediciones TALASA, Madrid, 2002

DOMENACH Hervé y PICOUET Michel, "Las Migraciones", Universidad Nacional de Córdova, Argentina, 1995

ESCUDERO GALANTE Francisco José, “Inmigrantes Magrebíes en Elche: Plan de Integración Social, Departamento de Sociología II, Universidad de Alicante, 2001

GOYCOECHEA Alba, Los imaginarios migratorios" (El caso ecuatoriano), Ediciones ABYA AYALA, Ecuador, 2.003, Pág. 13 
GOYCOECHEA Alba y RAMIREZ Gallegos Franklin, "Se fue, ¿a volver?, (Imaginarios, familia y redes sociales en la migración ecuatoriana a España (1.997 2.00), en Revista ICONOS N. 14, Agosto del 2.002, Pág. 39

MALGASINI Graciela, "Cruzando Fronteras", (Migración en el sistema), Editorial Fundación Hogar del Empleado, Madrid, 1998

MANZANO BILBAO César, "El grito del otro: arqueología de la marginación racial", Editorial Tecnos, Madrid, 1999

MARTINEZ VEIGA Ubaldo, "Las integración social de los inmigrantes extranjeros en España", Editorial Trotta, Madrid, 1997

MAS Francesc, "Rompiendo Fronteras" (Una visión positiva de la migración), Ediciones Octaedro, Barcelona, Pág. 54.

PATIÑO y PESANTEZ, "La migración internacional: relatada e interpretada por los jóvenes en el país de origen y de destino" Studi Emigrazione, Revista Trimestral del Centro Studi Emigrazione Roma, Unione Stampa Periódica Italiana, 2.004.

PEÑA Francisco, "Mujeres indocumentadas: Redes sociales, Marginalidad y Pobreza", en Mujeres, Género y Desarrollo, GONZALES María Arcelia y otros, Morevallado Editores, México, 1.998

PORTES Alejandro y BOROCZ Jozsef, "Inmigración contemporánea: perspectivas teóricas sobre sus determinantes y modos de acceso", ALFOZ, Madrid, 1999

Revista Internacional de Ciencias Sociales, "Las migraciones internacionales 2002", N. 165, Editorial Serim Timur, Septiembre del 2000

SIISE, "Los indicadores sociales. La migración internacional reciente: algunos interrogantes", Artículo publicado en la Revista Gestión N. 90 de diciembre del 2.001.

SUTCLIFFE Bob, "Nacido en otra parte" (Un ensayo sobre la migración internacional, el desarrollo y la equidad), Marra Publicidad, Bilbao, 1998

\section{ANEXO I}

\begin{tabular}{|c|c|c|c|c|c|c|c|c|c|c|c|}
\hline \multicolumn{2}{|c|}{ ORIGEN } & \multicolumn{2}{|c|}{ DESTINO } & \multicolumn{2}{|c|}{$\begin{array}{l}\text { TIEMPO DE } \\
\text { MIGRACIÓN }\end{array}$} & \multicolumn{2}{|c|}{$\begin{array}{l}\text { SITUACIÓN } \\
\text { LEGAL }\end{array}$} & \multicolumn{2}{|l|}{ MERCADO LABORAL } & \multicolumn{2}{|l|}{ INSTRUCCIÓN } \\
\hline Azuay & 8 & Madrid & 6 & $1-2$ & 3 & Regular & 15 & Agricultura & 5 & Primaria & 8 \\
\hline Cañar & 6 & Barcelona & 5 & $3-4$ & 9 & Irregular & 5 & Construcción & 4 & Secundaria & 4 \\
\hline Loja & 6 & Jumilla & 4 & $5-6$ & 6 & & & Cuidado niños y ancianos & 4 & Secundaria incompleta & 1 \\
\hline & & Murcia & 4 & $7-8$ & 2 & & & Empleadas domésticas & 3 & Superior & 3 \\
\hline & & Alicante & 1 & & & & & Empresas & 2 & Superior incompleta & 4 \\
\hline & & & & & & & & Venta en almacén & 1 & & \\
\hline & & & & & & & & Carpintería & 1 & & \\
\hline
\end{tabular}

